\title{
Spatial pyramidal cross correlation for particle image velocimetry
}

\author{
WANG HongPing ${ }^{1,2}$, WU Peng ${ }^{3}$, GAO Qi ${ }^{1 *}$, WANG JianJie ${ }^{1}$ \& WANG JinJun ${ }^{1}$ \\ ${ }^{1}$ Key Laboratory of Fluid Mechanics, Ministry of Education, Beihang University, Beijing 100083, China; \\ ${ }^{2}$ State Key Laboratory of Nonlinear Mechanics, Institute of Mechanics, Chinese Academy of Sciences, Beijing 100190, China; \\ ${ }^{3}$ Artificial Organ Technology Laboratory, Biomanufacturing Centre, School of Mechanical and Electrical Engineering, Soochow University, \\ Suzhou 215006, China
}

Received October 9, 2017; accepted January 9, 2018; published online February 10, 2018

\begin{abstract}
A spatial pyramidal cross-correlation based on interrogation area sub-division is introduced to improve the measurement resolution in particle image velocimetry (PIV). The high-resolution velocity can be achieved with a velocity prediction model via coarse cross-correlation. The prediction formula is deduced from the frequency response of the moving average (MA). The performance of this method was assessed using synthetically generated images of sinusoidal shear flow, two-dimensional vortical cellular flow, and homogeneous turbulence. A real PIV experiment of turbulent boundary layer was used to evaluate the new method. The results indicate that the spatial pyramid cross-correlation can robustly increase the spatial resolution.
\end{abstract}

particle image velocimetry (PIV), cross correlation, spatial resolution, moving average

Citation: Wang H P, Wu P, Gao Q, et al. Spatial pyramidal cross correlation for particle image velocimetry. Sci China Tech Sci, 2018, 61: 867-878, https://doi. org/10.1007/s11431-017-9191-3

\section{Introduction}

The flow velocity field can be obtained using particle image velocimetry (PIV) from the maximum peak of the correlation map over the interrogation windows [1]. Due to the inherent algorithm used in PIV, the obtained velocity is filtered according to the size of the interrogation windows (IW), which can be simply modeled using a moving average (MA) [2]. The modulation caused by MA can reduce the accuracy of the velocity obtained by cross-correlation (CC). The spatial resolution can be improved by reducing the size of IW. However, the signal-to-noise ratio (SNR) drops dramatically when the number of image particle pairs decreases to below ten in a small IW [1]. To overcome this limitation, iterative optimization with image deformation [3-5] was developed for flow field with large velocity gradients. The iterative process can be schematically described as multi-grid analysis

*Corresponding author (email: qigao@buaa.edu.cn) and iterative analysis [6]. The effect of predictor-corrector filtering on the stability and spatial resolution of iterative PIV interrogation was investigated theoretically and numerically by Schrijer and Scarano [6]. Although the accuracy is improved by iterative analysis with image deformation, the spatial resolution is still limited by the IW because the frequency response is negative when the size of the IW is larger than the local wavelength of the velocity.

Another improvement to the spatial resolution is to change the frequency response of the $\mathrm{CC}$ by weighting the interrogation window [2]. Adel and Riethmuller [7] developed a hybrid PIV-PTV method that combines the advantages of the robustness of PIV and the spatial resolution of particle tracking velocimetry (PTV). According to Scarano [8], the error of the MA depends on the local curvature radius of the displacement distribution, and it increases with the square of the window size. Thus, a super-resolution velocity field can be obtained by directly measuring the local second derivatives of the displacement distribution over the IW [8]. 
Complex approaches with spatial adaptivity according to the image properties and flow conditions were also introduced to overcome the limits of conventional PIV algorithms. In the adaptive image interrogation method proposed by Theunissen et al. [9], the location, number, and size of the IW were adaptively optimized according to the seeding density and velocity fluctuations. Novara et al. [10] introduced a method to adapt to the shape and orientation of interrogation volumes to a 3D-PIV motion analysis, which was a 3D extension of previous concepts using 2D analysis [11]. However, as commented by Theunissen et al. [12], the adaptation of the cross-correlation windows based on the instantaneous velocity fields is prone to error propagation due to the poor accuracy of the second order derivatives.

In PIV processing, improving the spatial resolution must consider the SNR of CC. For time-resolved PIV, the interrogation size can be significantly reduced by increasing the number of image pairs for long sequences $[13,14]$. Sciacchitano et al. [15] proposed a novel approach, the multiframe pyramid correlation, which combines the correlation maps of different temporal separations to enhance the SNR and precision. The correlation based correction (CBC) method proposed by Hart [16] applies an element-by-element multiplication of correlation tables calculated from adjacent regions to eliminate the stochastic correlation values during PIV processing. This method can significantly increase the SNR of CC. While, it seemed that the accuracy was not adequate [8]. Unlike the traditional outlier detection and elimination methods [17-19], the CBC method can remove the noise from the correlation plane.

In this work, a new spatial pyramid cross-correlation method was investigated to improve the spatial resolution in a robust manner. In contrast to the multi-frame pyramid correlation for time-resolved PIV [15], this method utilizes the multi-resolution correlation map of only one image pair to enhance the SNR. One-dimensional (1D) sinusoidal shear displacement, two-dimensional (2D) vortical cellular flow, and homogeneous turbulence were considered to assess the performance of the pyramidal CC. Moreover, a turbulent boundary layer (TBL) experiment was utilized to evaluate the method.

\section{Multi-spatial-resolution pyramidal cross- correlation}

\subsection{Cross-correlation based on subdivision of inter- rogation area}

The $\mathrm{CC}$ without normalization is expressed in the equation as

$C_{x, y}=\sum_{i=0}^{M} \sum_{j=0}^{N} f(i, j) \cdot g(i+x, j+y)$,

where the parameters $f(i, j)$ and $g(i, j)$ represent the fluctu- ating grey intensity indexed by $i$ and $j$ over the IW, and $f(i, j)$ is obtained from the particle images $I_{\mathrm{A}}, g(i, j)$ is from the particle images $I_{\mathrm{B}}$. The parameters $x$ and $y$ denote the shifted displacement where the CC is calculated. In this study, the size of $f$ is $M \times N$ and the dimension of $g$ is larger than $f$. It is reasonable to make a hypothesis that the particles is uniformly distributed over the measurement domain. Therefore, the normalization of the coefficient is not necessary. The coefficient map is obtained by shifting the smaller $f$ of image $I_{\mathrm{A}}$ over the $g$ of image $I_{\mathrm{B}}$. Then, the displacement can be evaluated by searching and fitting the peak of the coefficient array.

Figure 1 shows the schematic diagram of CC. The dotted lines denote the IW as $f$, and the solid lines denote the search region as $g$. As shown in the figure, the size of the final CC map is $(2 L+1,2 L+1)$. If we divided the IW into many subIWs with same size (as presented by the purpose dotted line), the size of the CC map of the sub-IW is also $(2 L+1,2 L+1)$, and it is easy to deduce that the $\mathrm{CC}$ of the full-IW is equivalent to the summation of the $\mathrm{CC}$ of the sub-IWs. This can be expressed as

$C_{\mathrm{f}}=\sum_{t=1}^{T} C_{\mathrm{s}}^{t}$,

where the subscripts of $f$ and $s$ indicate the full-IW and subIW. The parameter $T$ is the total number of sub-IWs indexed by superscript $t$. Ignoring the influence of the noise, every coefficient map of the sub-IW contains a peak corresponding to the local velocity, thus, eq. (2) means that the velocity obtained from full-IW is the average of the sub-IWs, which is very similar to the MA filter $[2,6]$.

One important advantage from the IW subdivision is to reduce the time cost of computation. As we known, a large overlap between adjacent vectors is applied in the PIV process to reduce the errors from interpolation and derivation in the velocity field. The overlap of the IW would increase the burden of computation since the $\mathrm{CC}$ of the overlap regions are repeatedly evaluated. A subdivision strategy is implemented according to the overlap factor. If a $50 \%$ overlap factor is applied in PIV, the full-IW is divided into $2 \times 2 \mathrm{sub}$ areas. Only the CC of sub-IW is calculated at each grid, instead of the full-IW. Thus, the computational cost can be reduced to $1 / 4$ under direct $\mathrm{CC}$. Additional operation is to sum the CC map of the sub-IWs according to eq. (2). While, for FFT-based CC, the cost reduction may be not so significant because FFT is already a fast and efficient algorithm. Figure 2 shows the computational time for full-IW and subIW at different overlap factors based on the FFT-based CC. The time cost can be reduced $30 \%$ and $23 \%$ for $50 \%$ and $75 \%$ overlap factor, respectively.

The velocity obtained using PIV is a spatial average of the real velocity over the IW. Owing to the low SNR in the small 


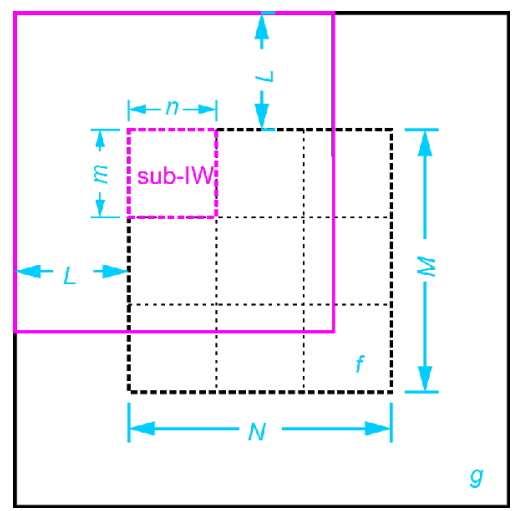

Figure 1 (Color online) Diagram of cross-correlation based on interrogation area subdivision. $N$ and $M$ denote the size of the IW, $L$ represents the search region over the $g$ of image $I_{\mathrm{B}}$. The lowercase letters $n$ and $m$ indicate the size of the sub-IW. All the parameters are in pixel scales.

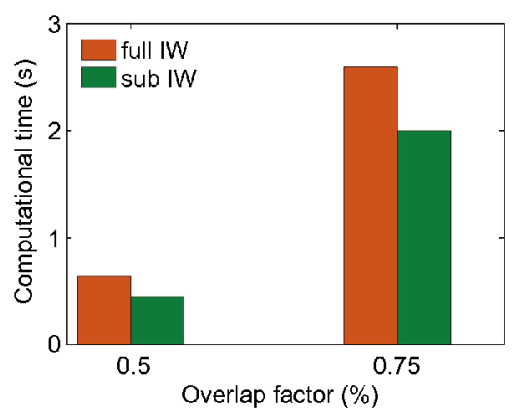

Figure 2 (Color online) The comparison of computational time between full-IW and sub-IW at different overlap factors, the FFT is adopted in the CC. The size of the test image is $512 \times 512$ pixels, and the size of the IW is $32 \times 32$ pixels. The computational time is the total time for processing a pair images.

sub-IW, $C_{\mathrm{s}}^{t}$ may contain pronounced random noise or outlier peaks. The summation of the adjacent sub-IWs can smooth out the noise and enhance the actual peak. Consequently, other proper and possible accumulation modes can also be introduced into eq. (2) to enhance the SNR of CC. The correlation based correction proposed by Hart [16] already used the multiplication of the correlation planes from one or more adjacent regions to eliminate anomalies. Moreover, in our opinion, the CC of full-IW can also be calculated by replacing the summation with median operation. Discussion of these methods goes beyond the scope of this study, and we still adopt the classical summation mode for this study.

\subsection{Local velocity prediction model}

A simple local velocity prediction model will be introduced in this part. This model will be used in pyramidal CC to predict the local velocity from the coarser information. The horizontal velocity component $u_{(x, y)}$ is given as eq. (3) by expanding the displacement distribution over the IW in the form of two order Taylor series.

$$
\begin{aligned}
u_{(x, y)}= & \left.u\right|_{\left(x_{0}, y_{0}\right)}+\left(\frac{\partial u}{\partial x}\right)_{\left(x_{0}, y_{0}\right)}\left(x-x_{0}\right)+\left(\frac{\partial u}{\partial y}\right)_{\left(x_{0}, y_{0}\right)}\left(y-y_{0}\right) \\
& +\left(\frac{\partial^{2} u}{2 \cdot \partial x^{2}}\right)_{\left(x_{0}, y_{0}\right)}\left(x-x_{0}\right)^{2}+\left(\frac{\partial^{2} u}{2 \cdot \partial y^{2}}\right)_{\left(x_{0}, y_{0}\right)}\left(y-y_{0}\right)^{2} \\
& +\left(\frac{\partial^{2} u}{\partial x \partial y}\right)_{\left(x_{0}, y_{0}\right)}\left(x-x_{0}\right)\left(y-y_{0}\right),
\end{aligned}
$$

where the parameter $\left(x_{0}, y_{0}\right)$ represents the center of the IW, and $(x, y)$ denotes the position over the IW. The expression of the vertical velocity component $v$ is similar to $u$. Since the velocity obtained by $\mathrm{CC}$ is similar to the velocity of MA filter, the velocity from MA filter with length $d$ (size of a square window) at $\left(x_{0}, y_{0}\right)$ is

$\bar{u}_{\left(x_{0}, y_{0}\right)}=\left.u\right|_{\left(x_{0}, y_{0}\right)}+\frac{d^{2}}{24} \cdot\left(\left(\frac{\partial^{2} u}{\partial x^{2}}\right)_{\left(x_{0}, y_{0}\right)}+\left(\frac{\partial^{2} u}{\partial y^{2}}\right)_{\left(x_{0}, y_{0}\right)}\right)$.

This result can also be found in the article by Scarano [8]. The MA result $\bar{u}$ only depends on the local real velocity, the second-order partial derivative and the filter window size. In order to figure out $\left.u\right|_{\left(x_{0}, y_{0}\right)}$, another MA filtering with different filter size is adopted to the velocity field. Therefore, $\left.u\right|_{\left(x_{0}, y_{0}\right)}$ can be predicted as

$\left.u_{\mathrm{p}}\right|_{\left(x_{0}, y_{0}\right)}=u_{d_{2}}+\frac{d_{2}^{2}}{d_{1}^{2}-d_{2}^{2}} \cdot\left(u_{d_{2}}-u_{d_{1}}\right)$,

where $u_{\mathrm{p}}$ is the predicted velocity at the center of the IW. The parameters $d_{1}, d_{2}$ is the window size of the MA filter, and $d_{1}>d_{2} . u_{d_{1}}, u_{d_{2}}$ is the velocity filtered by the different filter size. According to eq. (5), the local real velocity can be predicted from the low resolution values. Figure 3 shows the predicted result from one-dimensional signal with variable wavelengths. The filter length $d_{1}$ and $d_{2}$ is 48 and 32, respectively. Note that wavelength is increased from 30 at $x=0$ to 50 at $x=200$. From this picture, the predicted signal shows a good agreement with the ground truth at large wavelength. The deviation between $u_{\mathrm{p}}$ and $u_{\text {real }}$ increases with decreasing wavelength. The prediction is much closer to the real velocity than other two MA results.

The accuracy of the prediction is relative to the filter size and local velocity wavelength (or the local velocity gradient), as can be seen in Figure 3. The accuracy of the prediction can be deduced from the simplified one-dimensional frequency response of MA. For a wave signal with wavelength $\lambda$, the result of MA with filter length $d$ is also given as

$u_{d}=\operatorname{sinc}\left(\frac{d}{\lambda}\right) \cdot u$,

where the coefficient $\operatorname{sinc}(d / \lambda)$ is the amplification of the wavelength (Figure 4). Combing the eq. (5) and eq. (6), the 


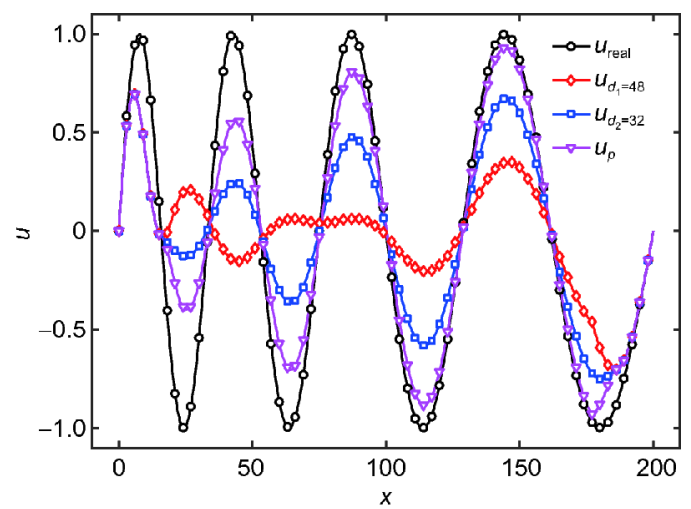

Figure 3 (Color online) One dimensional example of the local velocity prediction model. The ground truth indicated by the line with circle markers is written as $u_{\text {real }}=\sin \left(\frac{2 \pi}{30+0.1 x} \cdot x\right)$, the wavelength $(30+0.1 x)$ increases with $x$, and $x$ is set to be an integer from 0 to 200 . The lines marked by diamonds and squares are the results of MA with different filter length, the diamond is for $d_{1}=48$ and the square is for $d_{2}=32$. The line with triangles represents the predicted value from the eq. (5).

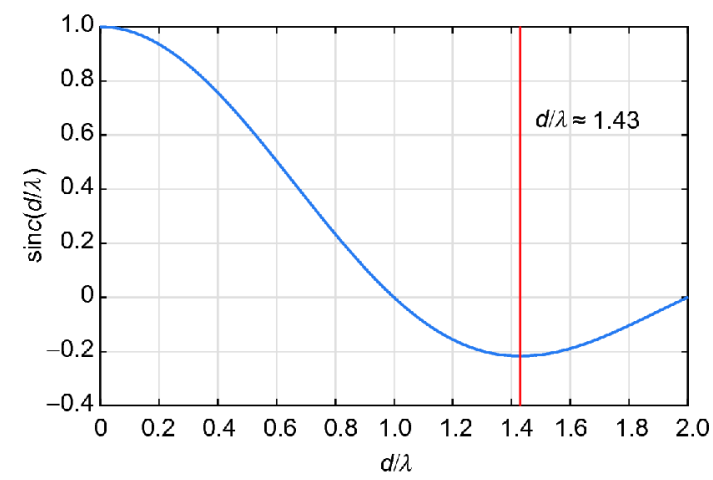

Figure 4 (Color online) Frequency response of MA [2,5,8]. The parameters $d$ and $\lambda$ represent the filter size and the wavelength of the wave signal, respectively. The vertical line indicates the local minimum at $d / \lambda \approx 1.43$.

predication formula can be rewritten as

$u_{\mathrm{p}}=\left(\left(\operatorname{sinc}\left(\frac{d_{2}}{\lambda}\right)+\frac{d_{2}^{2}}{d_{1}^{2}-d_{2}^{2}} \cdot\left(\operatorname{sinc}\left(\frac{d_{2}}{\lambda}\right)-\operatorname{sinc}\left(\frac{d_{1}}{\lambda}\right)\right)\right)\right) \cdot u$.

If $d_{2} / \lambda<d_{1} / \lambda<1.43$, the modulation coefficient of $u_{\mathrm{p}}$ is always larger than $\operatorname{sinc}\left(d_{2} / \lambda\right)$, which means the prediction is more accurate than the MA filtering result $u_{d_{2}}$.

\subsection{Spatial pyramidal cross-correlation}

The local velocity prediction model based on MA can be used in $\mathrm{CC}$, even though the frequency response of $\mathrm{CC}$ for particle images is somehow more complex than that of MA [2]. With this prediction model, a multi-resolution pyramidal architecture is developed based on interrogation area subdivision. According to eq. (2), the $\mathrm{CC}$ of the full-IW $\left(\mathrm{C}_{\mathrm{f}}\right)$ can be estimated as the summation of the $\mathrm{CC}$ of the sub-IW $\left(C_{\mathrm{s}}\right)$.
Therefore, increasing the number of sub-IWs can increase the size of the full-IW, consequently reduce the resolution of the CC. To simplify the discussion and expression, a square sub-IW is adopted, and its size is denoted as $m \times m$ pixels. Empirically, the size of sub-IW should guarantee that there is at least one particle in the sub-IW. A full-IW is expanded using multiple sub-IWs, whose number is $M \times M$, which means the size of the full-IW is $(M \times m) \times(M \times m)$ pixels. The idea of multi-resolution pyramidal $\mathrm{CC}$ is shown in Figure 5. In this figure, the $l$ represents the current layer of the pyramid, and the $M_{l}$ is the number of sub-IWs at the $l$ layer. The parameter $M_{l}$ is given as an odd number for the convenience to locate the center of the $\mathrm{CC}$, and it can be easily calculated as

$M_{l}=2 \times\left(l_{\max }-l\right)+1$,

where $l_{\max }$ is the total number of layers in the pyramid.

The local velocity at the $l$ layer can be predicted from the $l-1$ and $l-2$ layer according to the velocity prediction model as shown in eq. (5). In this part, we use $\mathbf{V}$ to present the $2 \mathrm{D}$ velocity vector $[u, v]$ of PIV. The expression for the prediction is written as

$\mathbf{V}_{\mathrm{p}}=\mathbf{V}_{l-1}+\frac{M_{l-1}^{2}}{M_{l-2}^{2}-M_{l-1}^{2}} \cdot\left(\mathbf{V}_{l-1}-\mathbf{V}_{l-2}\right)$,

where $\mathbf{V}_{l-1}$ and $\mathbf{V}_{l-2}$ is the coarse velocity at the $l-1$ and $l-2$ layer, and $\mathbf{V}_{\mathrm{p}}$ is the predicted velocity at the $l$ layer.

The CC at $l$ layer is refined to suppress the noise based on the predicted velocity. At $l$ layer, the CC map contains more random noise because of the smaller IW size. However, the predicted displacement can be used to enhance the signal of $\mathrm{CC}$ in the current layer. The pyramid correlation algorithm is realized by subpixel-shifting the coarse $\mathrm{CC}$ plane to the peaks of the current $\mathrm{CC}$ plane based on the difference in displacement. The $C_{l}$ represents the $\mathrm{CC}$ of the $l$ th layer, and the distance of the correlation peaks between $l$ th layer and $(l-k)$ th layer can be approximated as $\mathbf{V}_{\mathrm{p}}-\mathbf{V}_{l-k}$. Note that velocity of current layer $\mathbf{V}_{l}$ is replaced with $\mathbf{V}_{\mathrm{p}}$. The CC map at $(l-k)$ th layer is shifted toward to the direction $\mathbf{V}_{\mathrm{p}}-\mathbf{V}_{l-k}$ using a linear interpolation. After shifting, the position of the correlation peaks at different layers is approximately equal to the predicted value $\mathbf{V}_{\mathrm{p}}$, and the new correlation matrix is denoted as $\vec{C}_{l-k}$ where $k$ is from 0 to $l-1$ and the symbol “ $\leftrightarrow$ ” represents the shifting operation. The enhanced CC $\left(\bar{C}_{l}\right)$ at current layer is realized by accumulating the shifted coarse $\mathrm{CC}$ as

$\bar{C}_{l}=\frac{1}{l} \sum_{k=0}^{k=l-1} \bar{C}_{l-k}$.

A brief description of procedures is given in Table 1. A 


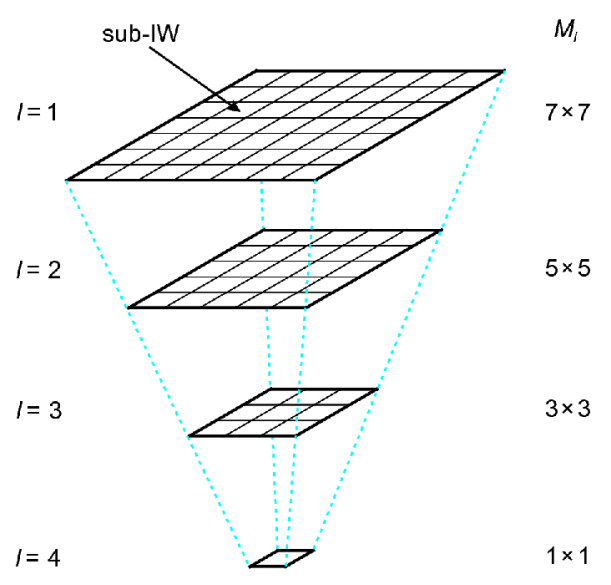

Figure 5 (Color online) Sketch of multi-resolution pyramidal cross-correlation.

new, more accurate, and robust displacement is obtained from the maximum peak of the enhanced $\mathrm{CC}$ plane because the random noise is suppressed by the coarse $\mathrm{CC}$.

\subsection{Iterative image deformation with spatial pyramidal $\mathrm{CC}$}

The pyramidal CC provides a robust method to calculate the displacement with high resolution. It is convenient and easy to apply pyramidal $\mathrm{CC}$ in the algorithm of iterative image deformation (IID). After estimating the displacement using pyramidal $\mathrm{CC}$, both of the $\mathrm{A}$ and $\mathrm{B}$ images are deformed to the center time according to the interpolated displacements with the center difference pattern. The displacement is validated using normalized median test and smoothed by $3 \times 3$ Gaussian filter. Linear and spline interpolations are applied to the images and displacements, respectively. The iterative analysis, where the grid nodes are fixed, is adopted to further improve the accuracy of the measurement. In this study, the iteration number of the image deformation is denoted by $n$.

\section{Numerical assessment}

The method's performance was assessed by statistically analyzing the synthetic images of sinusoidal shear flow, 2D vortical cellular flow, and homogeneous turbulence. The particle images were generated according to the framework proposed by Lecordier and Westerweel [20] and Pan et al. [21]. The CCD resolution was $512 \times 512$ pixels, and the ratio of the sensitive area of one pixel to the pixel pitch was fixed to one. The intensity of the seeding particles followed a standard Gaussian distribution. The intensity level of the particle images was obtained by the $2 \mathrm{D}$ integration of the Gaussian function. The mean diameter of the particle image was two pixels with a zero pixel standard deviation [22]. Gaussian white noise with a different variance was added to every pixel. The noise level was set to the ratio of the standard deviation of the noise intensity to the maximum brightness. The noise level was from 0 to $40 \%$ with a uniform interval of $5 \%$. The seeding density of particles was 0.08 particles per pixel (ppp), which returned about 20 particles within an IW of $16 \times 16$ pixels.

The present pyramidal method is compared with the iterative window deformation technique, which herein will be referred to as conventional PIV. To statistically evaluate the performance of different methods, the root mean square error (RMSE) of the $u$-component are calculated according to the formula [23]:

$u_{\mathrm{RMSE}}=\sqrt{\frac{1}{N} \sum_{\mathbf{x} \in \Omega}\left\|u_{\mathrm{c}}(\mathbf{x})-u_{\mathrm{e}}(\mathbf{x})\right\|^{2}}$,

where the parameter $N$ is the total number of grid nodes in the velocity field, the parameters $u_{\mathrm{c}}(\mathbf{x})$ and $u_{\mathrm{e}}(\mathbf{x})$ represent the computed and exact velocity respectively, the parameter $\boldsymbol{\Omega}$ represents the domain of the velocity field.

\subsection{One-dimensional sinusoidal shear displacement}

The one-dimensional simulation was performed using a sinusoidal displacement with wavelength $\lambda=40$ pixels and amplitude $U_{0}=2$ pixels [8]. To test the high-resolution performance of the pyramidal CC, a small sub-IW of $4 \times 4$ pixels with 5 pyramidal layers $\left(l_{\max }=5\right)$ was adopted to calculate the displacement distribution. The size of the full-IW of the first

Table 1 Algorithm for the pyramidal cross-correlation

\footnotetext{
Algorithm

a Calculate the $\mathrm{CC}$ of the sub-IW.

$\mathrm{b}$ Calculate the displacement distribution of the coarse $\mathrm{CC}$ of the $l$ th layer.

c Validate the velocity field using a normalized median test [19] or other effective outlier detection methods.

d If $l \geq 3$ :

d1 Predict the displacement based on eq. (9).

d2 Enhance the CC map of the current layer based on eq. (10).

d3 Estimate the new displacement $d_{l}$ from the enhanced $\bar{C}_{l}$ and validate the new velocity field.

d4 Increase $l$ by 1 until $l$ reaches its maximum value.
} 
layer is 36 pixels, and the size of the last layer is 4 pixels. This configuration yielded a velocity of $128 \times 128$ gird points. Note that only a single pass PIV process without iteration was adopted in this case.

Figure 6 shows the correlation maps obtained by the pyramidal $\mathrm{CC}$ and the conventional $\mathrm{CC}$ at the same node in different layers without considering the noise. The $\mathrm{CC}$ of the first two layers is identical, and the enhancement starts at the third layer. For conventional CC, the SNR of the CC decreases with an increase in $l$ owing to a reduction in the size of the full-IW. When the size of IW is equal to four pixels $(l=5)$, the $\mathrm{CC}$ is almost contaminated by noise. However, the pyramidal CC still generates a clear peak in the correlation plane with a higher SNR. The CC was normalized by its maximum value, and the high correlated distribution is elongated along the X-direction because of the high displacement gradient. As shown in Figure 6, the pyramidal CC can achieve a high-resolution in a more robust manner.

The velocity profile calculated by the pyramidal CC is presented in Figure 7(a). The $u$-component is averaged along the $X$-direction, and the color represents the layer of the pyramid, except for the actual simulated data. With increasing $l$, the peaks of the wave gradually approach two pixels, which indicates that the modulation effect of the MA is reduced by decreasing the size of the IW. In order to test the stability of the pyramidal CC on noise, Gaussian white noises with noise levels of $20 \%$ and $40 \%$ were added to the synthetic images. The single-pass displacement was validated using a normalized median test [19] without smoothing, and the error was estimated according to eq. (11). Figure 7(b) shows the RMSE of the $u$-component as a function of the pyramidal layers and noise level. Even though the size of the IW is only four pixels at the fifth layer, the error is reduced to approximately half of that in the first layer. The pyramidal CC is stable although it is slightly affected by the image noise.

\subsection{Two-dimensional vortical cellular flow}

The $2 \mathrm{D}$ performance was assessed using the simulated vor-
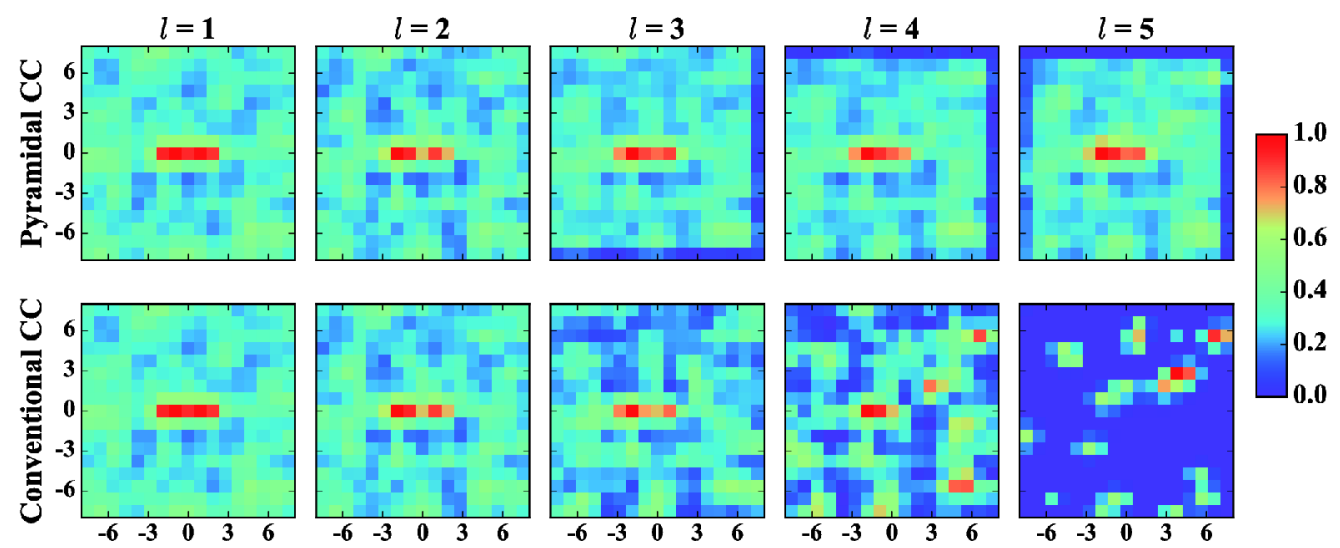

Figure 6 (Color online) Comparison of the CC planes between the conventional method and pyramidal method in different pyramidal layers.
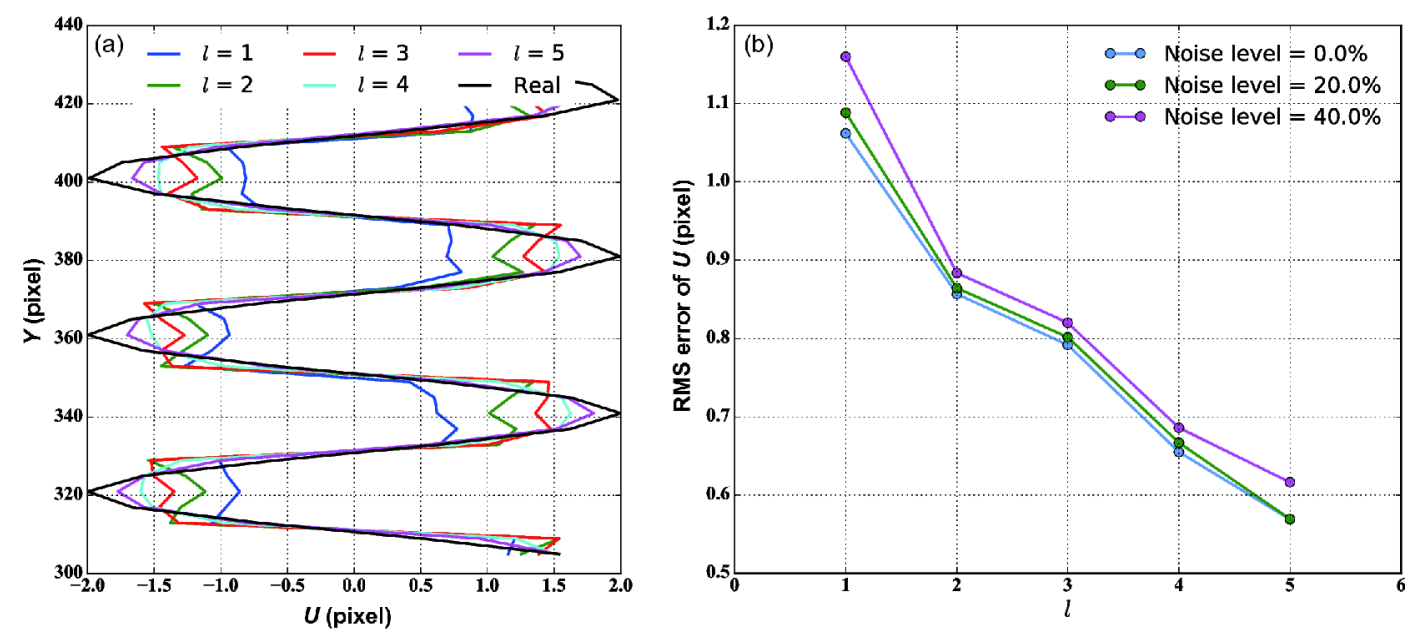

Figure 7 (Color online) (a) Velocity profiles calculated by the present pyramidal CC and (b) RMS error of $u$ as a function of the layers $l$ and the noise level. 
tical cellular flow with a varying wavelength $[21,24]$. The maximum of the $u$-component was set to a fixed value of three pixels while the wavelength decreased linearly from 130 pixels to 30 pixels along the $X$ - and $Y$-axes. The $v$ component has the same configuration as $u$. The expression of the vortical cellular flows is given as

$$
\begin{aligned}
& u=u_{\max } \cos \left[\frac{2 \pi k_{x}}{\left(130-0.2 k_{x}\right)}\right] \sin \left[\frac{2 \pi k_{y}}{\left(130-0.2 k_{y}\right)}\right], \\
& v=v_{\max } \sin \left[\frac{2 \pi k_{x}}{\left(130-0.2 k_{x}\right)}\right] \cos \left[\frac{2 \pi k_{y}}{\left(130-0.2 k_{y}\right)}\right],
\end{aligned}
$$

where both of $u_{\max }$ and $v_{\max }$ are equal to three pixels. The parameters $k_{x}$ and $k_{y}$ represent the index of the images along the $X$-axis and $Y$-axis, respectively. The denominator $\left(130-0.2 k_{x}\right.$ or $\left.130-0.2 k_{y}\right)$ represents the varying wavelength. As shown in Figure 8(a), the size of the 2D-vortex decreases, and the magnitude of vorticity increases from the top-left corner to the bottom-right corner, owing to the reduction in the wavelength of the velocity components. This flow field contains a series of vortices, and their intensities are inversely related to their scales.

The remainder of Figure 8 shows the comparison between the results calculated from conventional PIV and pyramidal PIV. Figure $8(\mathrm{~b})$ shows the spatial distribution of the velocity and vortices computed by conventional PIV with an IW=32 pixels. Figure 8(b1) shows one-pass iteration without image deformation, and (b2) shows a three-pass iteration with image deformation. Figure 8(c) shows the spatial distribution computed by pyramidal PIV with a sub-IW=4 pixels and $l_{\max }=5$. According to eq. (8), the size of the IW in first layer is $36 \times 36$ pixels. Figure $8(\mathrm{c} 1)$ shows a one-pass iteration without image deformation and Figure 8(c2) shows a threepass iteration with image deformation. In the Figure 8(b), the conventional PIV is unable to estimate the displacement at the bottom-right corner, owing to the modulation effect of the IW on the small-scale vortices. Even though the image is deformed according to the predictor, the displacement could not be recovered from the previous wrong information. However, at the upper-left corner of the large vortices, the IID can significantly reduce the error introduced by the modulation effect of a large IW. In the Figure 8(c), the hierarchical vortices could be well resolved even without applying IID, as shown in Figure 8(c1). The smallest structures at the bottom-right corner are not identical to the theoretical pattern in Figure 8(a). However, this situation is

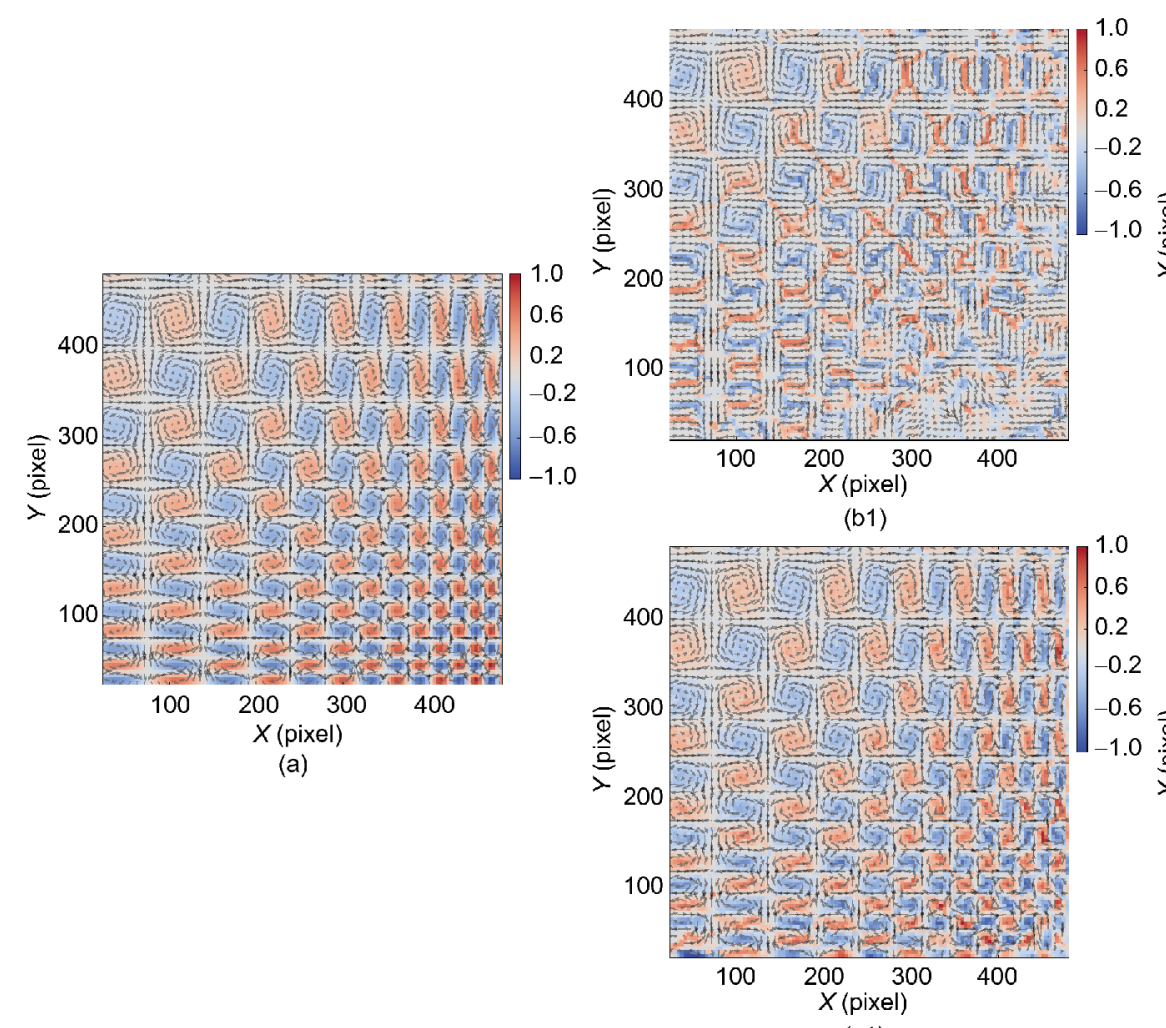

(c1)

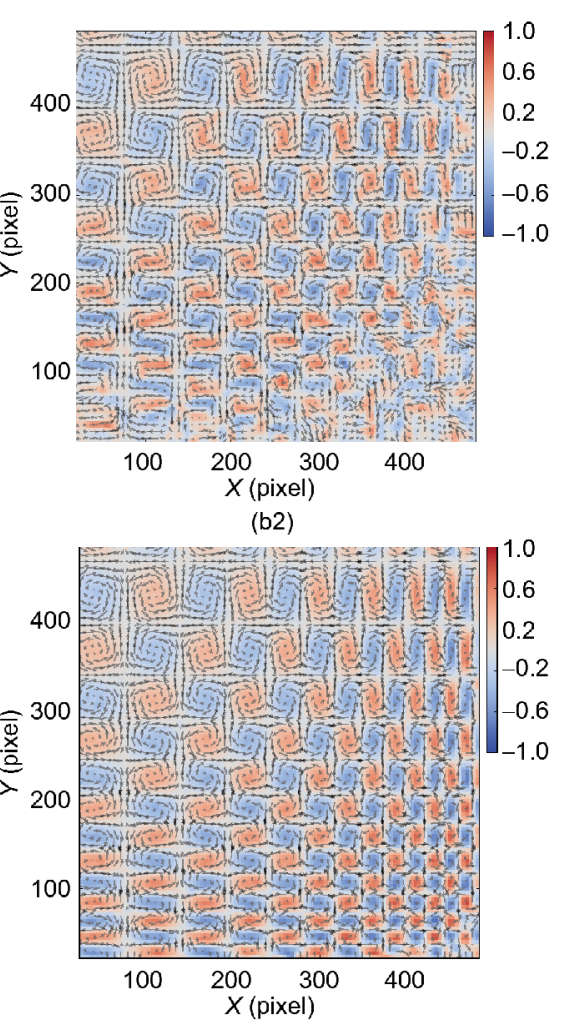

(c2)

Figure 8 (Color online) Spatial distribution of velocity and the vortices in the multi-vortex flow. (a) The imposed particle image displacement (the vectors) and the corresponding vortex (the contour). The vectors are down-sampled by a spacing of 8 to give a clear picture. (b) The distribution computed by conventional PIV of IW=32 pixels: (b1) a one-pass iteration without image deformation; (b2) a three-pass iteration with image deformation. (c) The distribution computed by pyramidal PIV of sub-IW=4 pixels and $l_{\max }=5$ : (c1) a one-pass iteration without image deformation; (c2) a three-pass iteration with image deformation. 
improved by combining pyramidal PIV and IID, as shown in Figure 8(c2). The conventional PIV can resolve the smallscale structures by reducing the size of the IW. However, the incremental outliers decreased the reliability of the measurement.

\subsection{Two-dimensional homogeneous turbulence}

Apart from the vortical cellular flow described in the previous section, the $2 \mathrm{D}$ homogenous and isotropic turbulent flow is adopted to evaluate the performance of the present method. The velocity data was downloaded from the Internet (http://fluid.irisa.fr/data-eng.htm) and was generated by the Cemagref team [25] in Rennes, France. One instantaneous field among the data is used to generate particle image pairs with a size of $512 \times 512$ pixels. The Gaussian white noise levels of $0 \%$ and $40 \%$ were added to the image pairs. The ppp is set to 0.15 to generate high quality images. In this test, a comparison between pyramidal PIV and conventional PIV is performed to check the resolution and accuracy. For the pyramidal PIV, two configurations are adopted. The size of the sub-IW is six pixels, and the $l_{\text {max }}$ is equal to four in the first configuration. The size of the sub-IW is four pixels and the $l_{\max }$ is equal to five in the second configuration. For conventional PIV, a series of configurations with different final IWs ( 4 to 48 pixels) are tested. The IID is used in both methods. Figure 9 displays the instantaneous vortex map (the top row) and error distribution (the bottom row) of the tested velocity field without artificial imaging noise. The maximum displacement is approximately about 3.5 pixels. The error vectors are calculated as the difference between the exact DNS data and the computed data. The vectors are downsampled to a space of 12 pixels to provide a clear display. Figure 9(a) and (b) shows the results of the conventional PIV with a final size of 6 and 30 pixels, and Figure 9(c) shows the result from the pyramidal PIV (first configuration). From the distributions of the error magnitude Figure 9(a2)-(c2), it is clear that the smaller IW (6 pixels) can introduce much higher noise than larger IW (30 pixels). The error of the latter always locate at high-velocity-gradient regions even though the images are deformed according to the velocity. The error of pyramid PIV (Figure 9(c)) is much smaller than that of conventional PIV.

Figure 10 shows the RMSE of the $u$-component as a function of the IW size. The errors of the conventional PIV are estimated for different IW sizes, and the errors of the

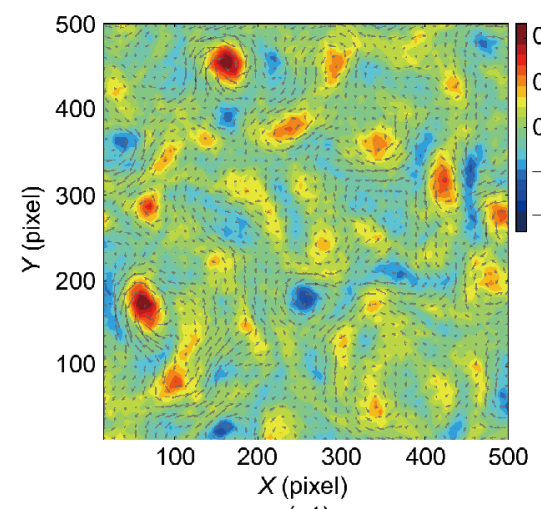

(a1)

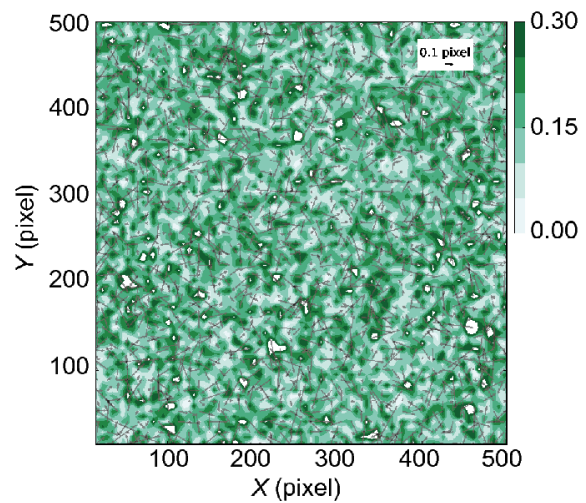

(a2)

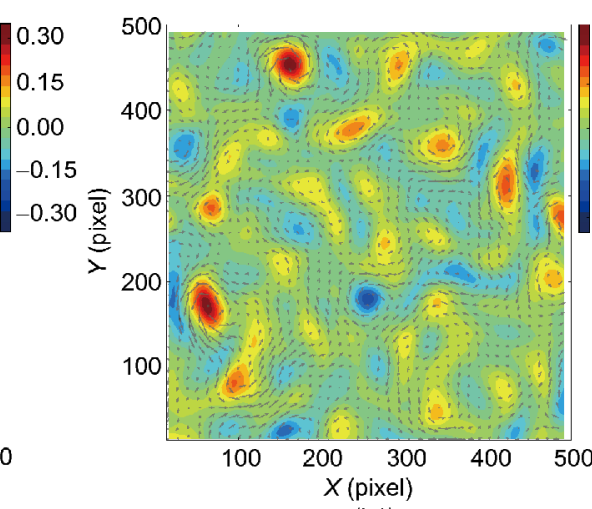

(b1)

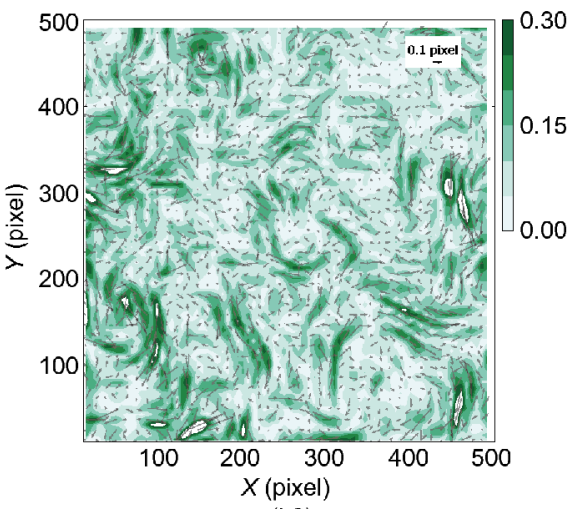

(b2)
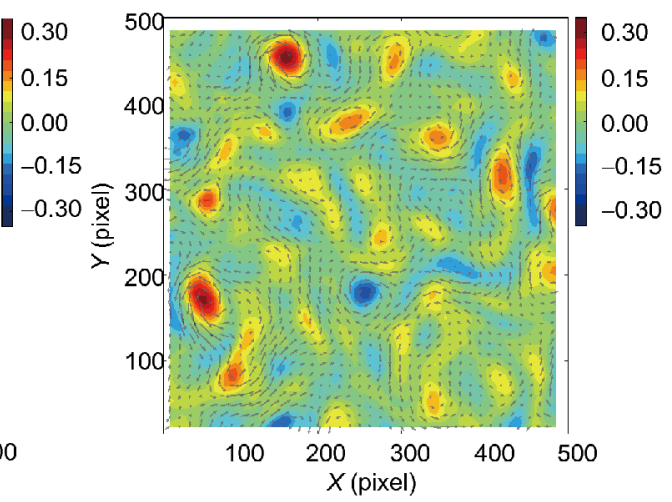

(c1)

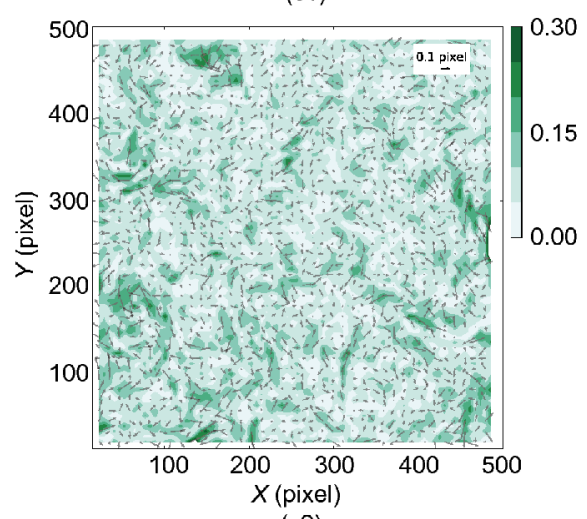

(c2)

Figure 9 (Color online) The instantaneous raw vortex field (the top row) and corresponding error distribution (the bottom row) of the test data without artificial imaging noise. (a) The result of the conventional PIV (final IW is 6 pixels); (b) the result of the conventional PIV (final IW is 30 pixels); (c) the result of the proposed pyramidal PIV with sub-IW $=6$ pixels and $l_{\max }=4$. The error vectors in (a2)-(c2) represent the difference between the exact DNS field and the measured velocities field. The filled contour maps display the vortex field and the error magnitude, respectively. All the computed velocities are raw data without smoothing. 
pyramidal PIV are estimated at each layer. The dashed lines with an $\mathrm{x}$ symbol and the solid lines represent the results of images with and without Gaussian white noise, respectively. In conventional PIV, the $u_{\text {RMSE }}$ presents a lowest value at the optimal size of the IW, which is the result of the balance between the effects of modulation and random noise. It is difficult to determine the optimal IW size in advance because it is infeasible to estimate the effects of seeding density and imaging noise. For pyramidal PIV, the errors in the first two layers with a large IW is identical to conventional PIV results. Different configurations of the pyramidal PIV show that the error almost monotonically decreases with a decrease in the size of the IW. The error in the pyramidal PIV at the smallest IW is much lower than that in conventional PIV at the same IW size and approximately equal to the lowest $u_{\text {RMSE }}$ of conventional PIV. This suggests that the pyramidal PIV is a robust and straightforward way to approach the optimized processing condition.

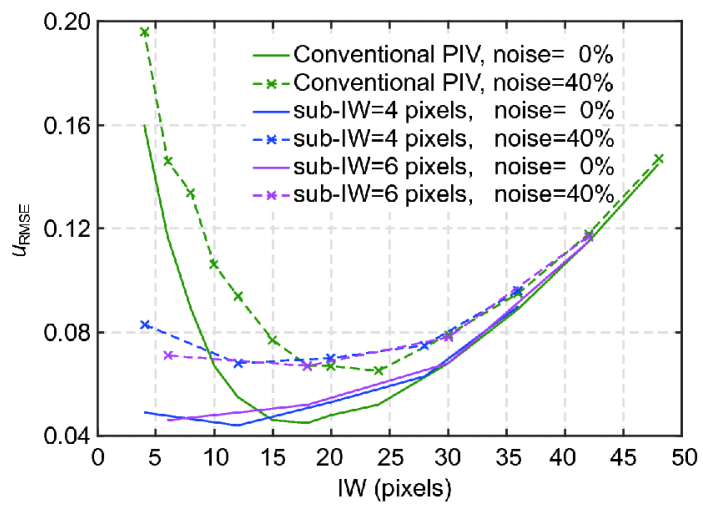

Figure 10 (Color online) The RMSE of $u$-component as a function of size of IW. The error of the pyramidal PIV is estimated at each layer. The solid lines are the results of the perfect images, while the dashed lines are obtained from the images with $40 \%$ Gaussian white noise.

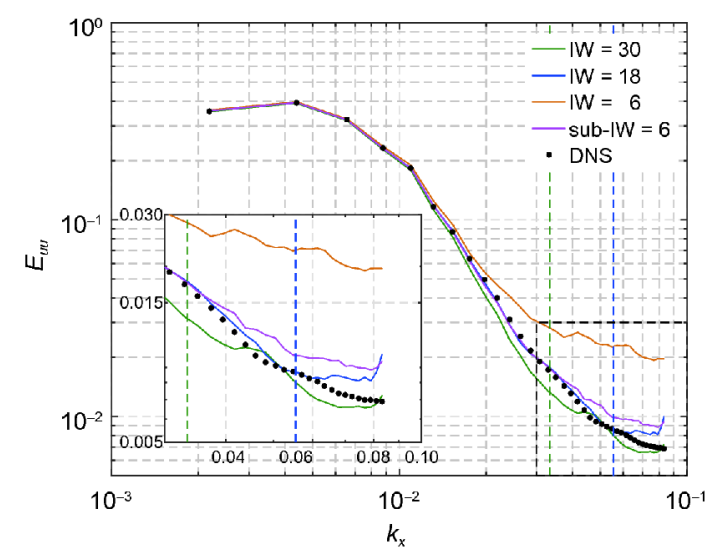

Figure 11 (Color online) Energy spectra of the $u$-component with a log$\log$ representation. The results were obtained from the images without noise. The dashed lines indicate the cutoff frequency of the IW. The parameter $k_{x}$ denotes the streamwise wavenumber. The details in the dashed box are enlarged within the small axis.
The details of the energy spectra of the $u$-component along the $X$-axis are presented in Figure 11. The results are obtained from the images without noise. The solid lines represent the results of conventional PIV and pyramidal method. The dashed lines indicate the cutoff frequency of the IW, and the energy spectra to the right side of the dashed lines are artificial for modulation effect. The parameter $k_{x}$ denotes the streamwise wavenumber. The spectrum of IW $=6$ pixels is contaminated by the random noise because of the small size of the IW. For a larger IW (IW=30 pixels), the energy is underestimated because of the strong modulation effect. The spectra of IW $=18$ and sub-IW $=6$ are both closer to the result of DNS, and the pyramidal PIV is slightly greater than IW=18 at high wavenumbers. The error of the pyramidal PIV approximately equal to the optimal IW in conventional PIV without image noise (also shown in Figure 10). It indicates that the pyramidal PIV can rechieve the optimal result of conventional PIV.

\section{Experimental assessment on turbulent boundary layer}

The pyramidal method was applied to resolve the near-wall velocity profile of the TBL under actual flow conditions. The experiment was conducted in a water tunnel at the Beihang University in China. A flat acrylic glass plate with dimensions of $14.4 \times 1 \mathrm{~m}^{2}$ was vertically mounted in the tunnel to generate the desired plate TBL. The distance between the plate and the sidewall was $0.6 \mathrm{~m}$, which was much larger than the thickness of the boundary layer. The turbulent flow was tripped by a spanwise attached tripwire. The tripwire had a diameter of $3 \mathrm{~mm}$ and was located $100 \mathrm{~mm}$ downstream of the leading edge. The measured plane was parallel with the wall, and its center was located $12 \mathrm{~m}$ from the leading edge of the plate. The experimental configuration is depicted in Figure 12. The near-wall flow was measured by a time-resolved 2D PIV system. A continuous $532 \mathrm{~nm}$ semiconductor laser with a thickness of approximately $1 \mathrm{~mm}$ was used to illuminate the tracer particles in the flow, whose mean diameter was approximately $20 \mu \mathrm{m}$. The laser was perpendicular to the plate and located at the center of the spanwise. The particle images $(2048 \times 2048$ pixels, 16 bits $)$ were recorded by a high-speed FASTCAM SA2 CMOS camera at a rate of $1000 \mathrm{~Hz}$. To get the near-wall velocity, an objective with a $180-\mathrm{mm}$ long-focus length was used, and the imaging region included the wall of the plate. The distance between the lens and the measured region $\left(36 \times 36 \mathrm{~mm}^{2}\right)$ was approximately $45 \mathrm{~cm}$. The given experimental settings yielded a high magnification of 55 pixels $/ \mathrm{mm}$, and the maximum displacement was approximately 16 pixels.

Note that the seeding density in the near-wall region was lower than that seen in the normal PIV. Therefore, the ad- 


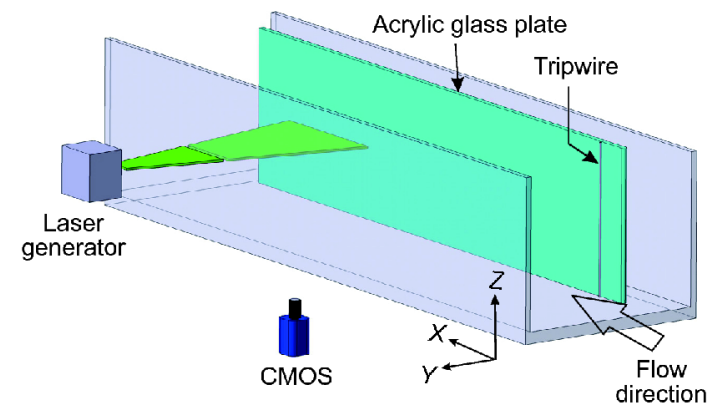

Figure 12 (Color online) Turbulent boundary layer experimental set-up.

jacent odd particle images were averaged to generate a new image series with higher ppp. Then, the images were preprocessed using historical minimum removal, sliding background removal, and a Gaussian smoothing $(3 \times 3$ kernel size). The ppp of the image was approximately 0.004 after preprocessing to remove noise (the peaks, whose intensity was larger than 5000 counts, were detected as particles). To perform a comparison between the conventional PIV method and the pyramidal method, 120 image pairs were randomly selected from the data sets. There was no significant reflection on the wall of the raw images.

The final size of the IW of the conventional PIV was set to $50 \times 50$ pixels, and the overlap factor was $80 \%$. There were approximately 10 particles in the IW. This configuration yielded a velocity field with $201 \times 201$ grid points. The outlier rate had reached $10 \%$ because of the low seeding density. For pyramidal PIV, five layers were used to resolve the velocity, the size of the first layer is $90 \times 90$ pixels, and the size of the sub-IW is $10 \times 10$ pixels. The coarse CC map will be used to refine the CC map of the sub-IWs. A three-pass image deformation analysis was utilized in both the methods. The intermediate velocity field was validated by a normalized median test [19] and smoothed by a $3 \times 3$ Gaussian kernel with a standard deviation of 0.6 , while the velocity field of the final iteration was validated without smoothing. Under these configurations, the computational cost of pyramidal $\mathrm{CC}$ was approximately about twice that of conventional CC.

The basic properties of the TBL were estimated by the measurement of laser Doppler velocimetry (LDV). The Musker profile method proposed by Kendall and Koochesfahani [26] was applied to fit the LDV data and estimate the basic properties of the TBL. The thickness of the boundary layer $\delta$ was $154.2 \mathrm{~mm}$, and the skin friction velocity $u_{\tau}$ was equal to $13.1 \mathrm{~mm} / \mathrm{s}$ when the free streamwise velocity $U_{\infty}$ was approximately $328.4 \mathrm{~mm} / \mathrm{s}$. The inner length scale $y^{*}$ was approximately $0.077 \mathrm{~mm}$. The Reynolds number based on momentum thickness $\left(R e_{\theta}\right)$ and skin friction velocity $\left(R e_{\tau}\right)$ were approximately 5039 and 2010, respectively.

A single snapshot of the TBL flow is shown in Figure 13. In Figure 13(a), the instantaneous velocity field and vorticity
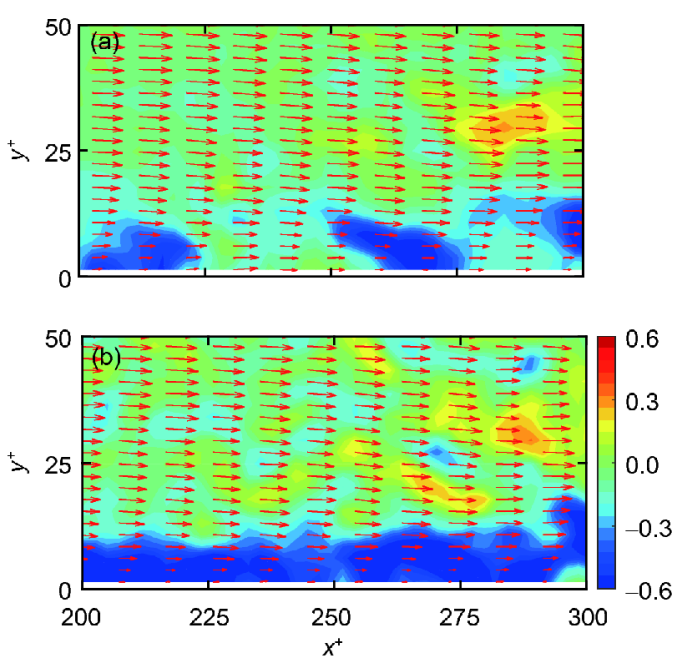

Figure 13 (Color online) Velocity vectors and vorticity contours for (a) conventional PIV and (b) pyramidal PIV in TBL experiment. Top: conventional PIV, bottom: pyramidal PIV.

field are obtained from conventional PIV with a window size of 50 pixels and an overlap factor of 80\%. In Figure 13(b), the results are carried out with 5 layers pyramidal PIV with sub-IW of 10 pixels. The negative vorticities in the inner layer are generated due to the non-slip condition. The shear layer in the bottom figure exhibits much more clearly than that in the top figure. The mean velocity profile and the fluctuating velocity profile obtained by different methods are given in Figure 14. The solid lines are the results of pyramidal PIV and conventional PIV, respectively. The $x$-markers represent the result of the LDV. As seen in the figure, obvious differences appear in the near-wall region $\left(y^{+}<10\right)$ for the mean and fluctuating profile owing to the high velocity gradient. The curve of pyramidal PIV is much closer to the LDV result than that of conventional PIV. For example, at $y^{+}=1.4$, the mean velocity of the present method and conventional method are 1.23 and 2.75 , respectively, while the fluctuating values are 1.62 and 2.45 , respectively. The results of the pyramidal PIV are much closer to the linear law for a viscous sublayer. The results of the pyramidal PIV and LDV are slightly lower than the linear law indicated by the dashed line, and the velocity fluctuation of pyramidal PIV is still higher than the LDV data. In the logarithmic region, the fluctuation of pyramidal PIV is higher than the conventional PIV, and the pyramid method is much closer to the LDV data, which indicates that the small-scale flow structures can be better resolved by the pyramidal PIV.

\section{Conclusions}

Limited by the particle density and image noise, the conventional PIV had to adopt a proper size of the IW to guar- 

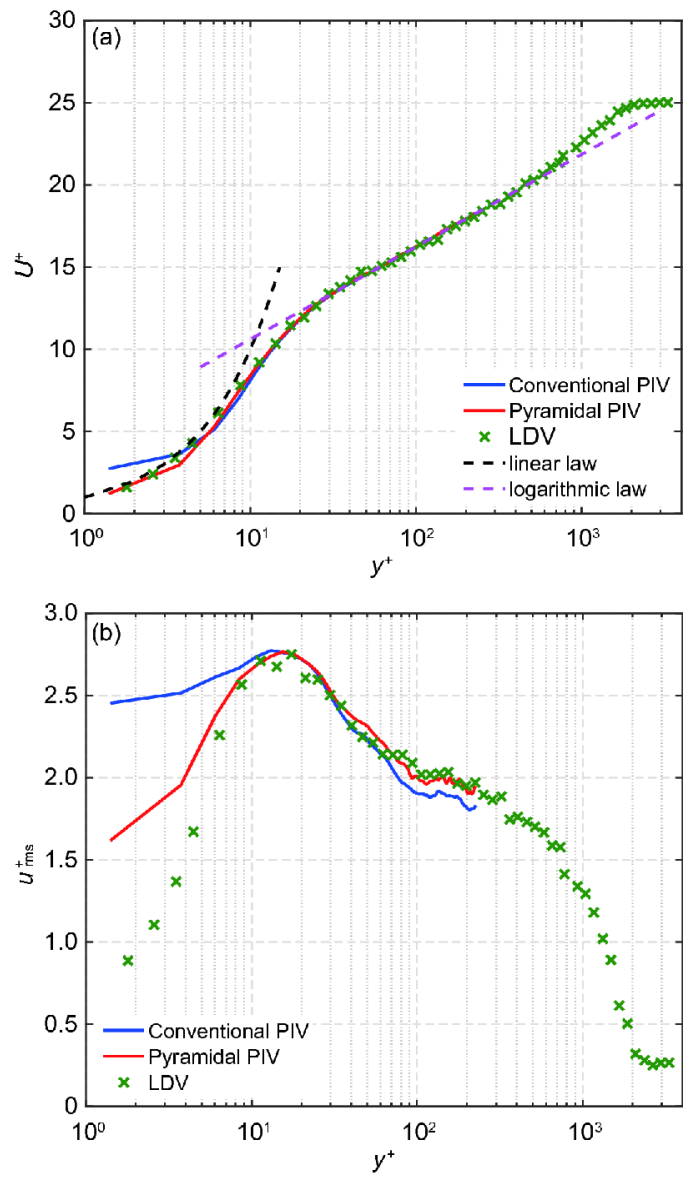

Figure 14 (Color online) Comparison between (a) mean velocity profiles and (b) the fluctuating velocity profiles of the TBL at $R e_{\tau} \approx 2010$.

antee accuracy and smoothness of the velocity. In practice, the optimal size of the IW is difficult to estimate in advance. The method presented in this study, spatial pyramidal cross correlation, could reduce the error to the lowest level with a small sub-IW size. Based on the frequency response of CC, the local velocity was predicted and corrected in a simple and robust manner. The key parameters are the size of the sub-IW $m$ and the total number of levels in the pyramid $l_{\max }$. The former is much smaller than the size of the IW in conventional PIV, however, it is also determined by the imaging noise and seeding density. It is suggested that there should be at least one particles in the sub-IW. The parameter $l_{\max }$ must be great than three and guarantee the robustness for the first two layers. Therefore, the sizes of the IWs in the first two layers are slightly larger than the size in conventional PIV. The performance of this method was assessed with three different flow types. The results indicated that the modulation effect of the MA can be reduced, and the small-scale structures can be resolved by this method. Specifically, when combined with iterative image deformation, this method further improved the accuracy to a level equal to that of the optimal conventional PIV. The application of this method to a TBL experiment showed that the near-wall velocity profile is better resolved by this method with time-consuming doubled versus the conventional PIV. The recently increasing interests in spatial-temporal energy spectra raise the requirement of space-time accurate velocity from PIV measurements [27-30]. The proposed method will be useful for this purpose. However, an increased noise level was also observed in the energy spectrum owing to the truncation of the predication and the noise of the images. This random noise can potentially be reduced by low-pass filtering.

This work was supported by the National Natural Science Foundation of China (Grant Nos. 11702302, 51406127 \& 11572331), the Fundamental Research Funds for Central Universities (YWF-16-JCTD-A-05) and the Natural Science Foundation of Jiangsu Province (Grant No. BK20140344).

1 Adrian R J. Particle-imaging techniques for experimental fluid mechanics. Annu Rev Fluid Mech, 1991, 23: 261-304

2 Nogueira J, Lecuona A, Rodríguez P A. Local field correction PIV: On the increase of accuracy of digital PIV systems. Exp Fluids, 1999, 27: $107-116$

3 Scarano F. Iterative image deformation methods in PIV. Meas Sci Technol, 2002, 13: R1-R19

4 Huang H T, Fiedler H E. Deformed particle image pattern matching in particle image velocimetry. Appl Sci Res, 1993, 51: 179-183

5 Scarano F, Riethmuller M L. Advances in iterative multigrid PIV image processing. Exp Fluids, 2000, 29: S051-S060

6 Schrijer F F J, Scarano F. Effect of predictor-corrector filtering on the stability and spatial resolution of iterative PIV interrogation. Exp Fluids, 2008, 45: 927-941

7 Stitou A, Riethmuller M L. Extension of PIV to super resolution using PTV. Meas Sci Technol, 2001, 12: 1398-1403

8 Scarano F. A super-resolution particle image velocimetry interrogation approach by means of velocity second derivatives correlation. Meas Sci Technol, 2004, 15: 475-486

9 Theunissen R, Scarano F, Riethmuller M L. An adaptive sampling and windowing interrogation method in PIV. Meas Sci Technol, 2007, 18: 275-287

10 Novara M, Ianiro A, Scarano F. Adaptive interrogation for 3D-PIV. Meas Sci Technol, 2013, 24: 024012

11 Stanislas M, Okamoto K, Kähler C J, et al. Main results of the second international PIV challenge. Exp Fluids, 2005, 39: 170-191

12 Theunissen R, Scarano F, Riethmuller M L. Spatially adaptive PIV interrogation based on data ensemble. Exp Fluids, 2010, 48: 875-887

13 Westerweel J, Geelhoed P F, Lindken R. Single-pixel resolution ensemble correlation for micro-PIV applications. Exp Fluids, 2004, 37: 375-384

14 Meinhart C D, Wereley S T, Santiago J G. A PIV algorithm for estimating time-averaged velocity fields. J Fluids Eng, 2000, 122: 285289

15 Sciacchitano A, Scarano F, Wieneke B. Multi-frame pyramid correlation for time-resolved PIV. Exp Fluids, 2012, 53: 1087-1105

16 Hart D P. PIV error correction. Exp Fluids, 2000, 29: 13-22

17 Shi S, Chen D. Enhancing particle image tracking performance with a sequential Monte Carlo method: The bootstrap filter. Flow Meas Instrum, 2011, 22: 190-200

18 Wang H P, Gao Q, Feng L H, et al. Proper orthogonal decomposition based outlier correction for PIV data. Exp Fluids, 2015, 56: 1-15

19 Westerweel J, Scarano F. Universal outlier detection for PIV data. Exp Fluids, 2005, 39: 1096-1100

20 Lecordier B, Westerweel J. The EUROPIV synthetic image generator 
(S.I.G.). In: Stanislas M, Westerweel J, Kompenhans J, Eds. Particle Image Velocimetry: Recent Improvements. Berlin Heidelberg: Springer, 2004. 145-161

21 Pan C, Xue D, Xu Y, et al. Evaluating the accuracy performance of Lucas-Kanade algorithm in the circumstance of PIV application. Sci China-Phys Mech Astron, 2015, 58: 104704

22 Sciacchitano A, Wieneke B, Scarano F. PIV uncertainty quantification by image matching. Meas Sci Technol, 2013, 24: 045302

23 Astarita T. Analysis of interpolation schemes for image deformation methods in PIV: Effect of noise on the accuracy and spatial resolution. Exp Fluids, 2006, 40: 977-987

24 Garcia D. A fast all-in-one method for automated post-processing of PIV data. Exp Fluids, 2011, 50: 1247-1259
25 Carlier B J, Wieneke B. Report 1 on production and diffusion of fluid mechanics images and data. Fluid project deliverable 1.2. http://www. fluid.irisa.fr, 2010

26 Kendall A, Koochesfahani M. A method for estimating wall friction in turbulent wall-bounded flows. Exp Fluids, 2008, 44: 773-780

27 He G W, Zhang J B. Elliptic model for space-time correlations in turbulent shear flows. Phys Rev E, 2006, 73: 055303

28 Zhao X, He G W. Space-time correlations of fluctuating velocities in turbulent shear flows. Phys Rev E, 2009, 79: 046316

29 de Kat R, Ganapathisubramani B. Frequency-wavenumber mapping in turbulent shear flows. J Fluid Mech, 2015, 783: 166-190

$30 \mathrm{He}$ G, Jin G, Yang Y. Space-time correlations and dynamic coupling in turbulent flows. Annu Rev Fluid Mech, 2017, 49: 51-70 